\title{
Safety and efficacy of enhanced recovery after surgery (ERAS) program after donor hepatectomy: a propensity-matched analysis
}

\author{
Sung Eun Park, Ho Joong Choi
}

Division of Hepatobiliary, Department of Surgery, The Catholic University of Korea, Seoul St. Mary's Hospital, Seoul, Korea

Background: Enhanced recovery after surgery (ERAS) programs have been shown to have benefits for various surgery, and recently, several studies have reported the advantages of the ERAS program in liver surgery. These achievements could be expected to improve the safety of donor hepatectomy, where the safety of donor is the most important, but studies are still lacking. The aim of this study was to assess the outcomes and benefits after implementation of an ERAS program on donor hepatectomy in living donor liver transplantation.

Methods: We analyzed perioperative outcomes on consecutive patients between January 2016 and October 2020 who underwent donor hepatectomy. Patients were divided into ERAS group and conventional surgery (CS) group. Propensity score matching (PSM) was used to define the independent effect of ERAS program on donor hepatectomy and 42 patients of ERAS group and 42 patients of CS group were enrolled.

Results: There were no significant differences in demographics characteristics between the two groups. The ERAS group was significantly lower in the rate of postoperative morbidity $(11.9 \%$ vs. $31 \%, P=0.033)$ and 90 -day readmission $(2.4 \%$ vs. $14.3 \%$, $\mathrm{P}=0.048$ ). The postoperative hospital days and the length of intensive care unit stay were significantly shorter for the ERAS group (10.2 vs. 11.4 days, $P=0.039$ ). Duration till first flatus and postoperative nausea and vomiting were significantly reduced in ERAS group. Pain control was better in ERAS (visual analogue scale: postoperative day 1, 13.5 vs. $2.7, \mathrm{P}<0.001$ ).

Conclusions: This study evaluated the impact of ERAS program approach on donor hepatectomy in living donor liver transplantation. ERAS programs applied to patients undergoing donor hepatectomy can safely and effectively reduce the incidence of complications, improve postoperative pain and bowel movement, accelerate patient recovery.

Corresponding author: Ho Joong Choi

E-mail: carin337@naver.com

(c) The Korean Society for Transplantation

This is an Open Access article distributed under the terms of the Creative Commons Attribution Non-Commercial License (http://creativecommons.org/licenses/by-nc/4.0/) which permits unrestricted non-commercial use, distribution, and reproduction in any medium, provided the original work is properly cited. 